\title{
Research on Non-standardized Evaluation Strategy of Course Learning Output Based on OBE
}

\author{
Jinbiao Zhang*, Jian Liu, Hongyun Zhang, Shoudong Chen, Xiangqi Xu \\ College of Mechanical Engineering, Tongling University, Tongling, China \\ ${ }^{*}$ Corresponding author. Email: zhangjb093@163.com
}

\begin{abstract}
In order to meet the need of Specialized Programmatic Accreditation of Engineering, a learning achievement evaluation strategy based on OBE is studied and practiced. Guided by learning output, a unit-achievement mapping matrix is constructed by Bloom's Taxonomy of Objectives, and a teaching model of course unit is construct on the basis of inquiry teaching method to carry out teaching activities, a non-standard assessment model on the whole process of course learning is put into effect, in which examination scores are analyzed by clustering algorithm and entropy weight method to timely synthesize the credible and efficient formative evaluation of learning output. The whole process non-standard evaluation strategy adheres to the OBE concept, effectively motivates the students to continue to improve, gradually realizes the successful learning and achieves the peak output.
\end{abstract}

Keywords: OBE, Learning output, Non standardization, Evaluation strategy.

\section{基于OBE理念的课程学习产出非标准化评价策略研究}

\author{
张金标*, 刘建，张红云，陈守东，徐向棋
}

铜陵学院机械工程学院, 铜陵, 中国

*通讯作者. 邮箱: zhangjb093@163.com

\section{中文摘要}

为适应工程教育专业认证需要, 研究并实践了一种基于 $\mathrm{OBE}$ 理念的课程学习成果评价策略。以学习产出为导 向逆向设计课程, 运用布卢姆目标分类法构建课程单元与学习产出映射矩阵, 逆向设计教学单元, 以探究式 教学法为策略构建单元教学运行模型, 使用探究式教学法开展教学活动, 对课程学习全过程进行非标准化考 核, 采用聚类算法和熵权算法综合分析考核成绩, 实现适时、可信、高效的学习产出形成性评价。全过程非 标准化评价策略秉承 OBE 理念, 有效激励学生持续改进, 逐步实现成功学习, 达成顶峰产出。

关键词: $O B E$, 学习产出, 非标准化, 评价策略

\section{1. 引言}

成果导向教育(Outcome-Based Education, OBE) 以其完整的理论体系和追求卓越的教育理念，近 20 年来已逐渐被《华盛顿协议》成员国相继采用，作 为工程教育专业认证标准的基础。 ${ }^{[1-3]} 2016$ 年 6 月我 国成为《华盛顿协议》正式成员以来, 国内高校正
逐步接受并实践 OBE 工程教育理念, 开展由课程导 向向成果导向转化的教育模式改革。国内版《工程 教育认证标准》秉承 OBE 教育理念, 并将其贯穿于 专业教育和认证全过程。OBE 以产出为导向设计并 实施教学过程, 让学生通过学习获得预期学习成 果。因此, 作为 OBE 教育模式重要环节之一, 学习 
成果(学习产出)评价尤为关键, 是花费教师精力最 多、最为复杂的环节。但国内高校在此领域内的研 究和实践相对较少, 成果较弱。[2]本文在对比分析 传统标准化学习考评与非标准化学习评价的基础 上, 研究并实践了一种课程学习全过程非标准化考 评策略, 基于 OBE 理念构建课程学习产出矩阵, 设 计单元教学运行框架, 实施探究式单元教学, 以学 习产出为导向全面综合评定学习全过程表现, 实现 差异化、个性化的学习产出评价, 达成 OBE 人人都 能成功学习、收获顶峰产出的教学目标。

\section{2. 课程学习评价模式}

\section{1. 传统标准化考评的不足}

学习评价就是评估学生阶段性学习后在知识增 长、能力提升等方面的表现即学习成果即学习产 出。传统的学习评价多数采用标准化考试, 根据考 试成绩评判学习成果高低。从 OBE 角度分析, 标准 化考试存在诸多问题:

(1) 测试题目统一、答案统一、评分标准统一, 不便于个性化学习评价, 不能开展多角度多元化评 价。[4-5]

(2)考核形式单一、内容僵化、评价主体唯一, 不 能多元化全面考核学生学习热情、过程表现、能力 和素养, 缺乏对学生的知识体系掌握程度及综合运 用和创新能力的评价, 评价效度较低、信度不高。 [6-7]

（3）标准化考试动态适时性差, 评价不及时, 不 利于学生持续改进逐步提高学习成果。

\section{2. 非标准化考评}

OBE 强调所有学生都是成功学习者, 经过一段时 间的有效学习都能有所收获有所提高。所以, 考核 学生学习效果, 评价学习产出就不能用标准化的、 相互竞争性的考核评价机制, 而应该根据学生个体 特点, 采用非标准化的考核体系进行个性化的、差 异化的学习评价, 适时开展考核评定, 及时反馈信 息, 让学生快速准确地掌握学习产出状况, 持续改 进, 自我竞争, 超越自我, 达成预期学习目标, 最 终收获学习顶峰成果。

越来越多的学者、教师接受并实践 OBE 理念, 与之相适应, 课程考核模式的改革也在深入发展。 近些年，多所高校教师先后就所授课程探索了不同 形式的非标准化考核模式, ${ }^{[1,4-8]}$ 大体上都是考核形 式多样化、内容多样化、评判主体多元化, 强调对 知识综合运用和创新能力的考核评价。非标准化考 核在很大程度上弥补了标准化考试的不足, 在一定 程度上反映了学生学习成效, 激发学生学习热情。 在此基础上, 本文探索实践的课程学习全过程非标 准化考评策略, 能更好地适应 OBE 课程教学模式的
需要。

\section{3. 课程学习全过程非标准化考评策略}

OBE 教育体系的底层是课程, 学生通过课程学习 达到毕业要求(即国内版工程教育专业认证中毕业要 求 12 条), 再经 5 年左右工程训练进而达成培养目 标, 成为高素质应用型专业人才。由此可见, 实践 OBE 教育理念的基础在于实施 OBE 课程教学模 式, 而课程教学目标即学生课程学习产出的达成是 OBE 教育目标的基石，而学习产出的达成性评价则 成为重中之重。基于 OBE 理论进行课程教学改革, 探索实践课程学习全过程非标准化考评是非常必要 的, 也是十分有意义的。

\section{1. 构建课程单元预期学习产出矩阵}

根据 OBE 思想, 教师(或教学团队)对所授课程首 先进行逆向设计, 以学生最终学习产出为起点, 明 晰课程单元 (Course Unit, CU) 对预期学习产出 (Intended Learning Outcome, ILO)的贡献程度, 设计 教学方案, 然后开展教学活动。本文借鉴教学团队 已有研究成果, 采用改进的布卢姆目标教学法(详见 文献[9])设计课程, 构建课程单元预期学习产出映射 矩阵。

依据专业课程体系的预期学习产出矩阵和课程教 学大纲, 分解所授课程的学习产出, 重组课程教学 内容和资源, 提炼单元知识和教学素材, 构建课程 教学单元, 设计教学单元与预期学习产出映射矩 阵。课程预期学习产出是课程体系为满足毕业要求 而设计的最低学习产出标准, 是全部学生经过有效 学习都应该达成的。课程单元的部分学习产出高于 课程体系的标准, 是为了满足学习能力较强、创新 意识较高的学生学习欲望而设计的, 激励他们努力 学习, 顶峰产出。这也是个性化教学、差异化评价 的需要。表 1 是材料成形及控制工程专业的专业课 程“材料轧制成形工程学”教学单元与预期学习产出 映射矩阵(部分), 矩阵中元素数值是依据布卢姆教学 目标分类法确定的“认知程度”, 表中最后一行是“材 料轧制成形工艺学”课程学习的标准预期产出。

\section{2. 设计单元教学运行模型框架}

为实施基于 $\mathrm{OBE}$ 的非标准化学习评价, 教学团 队借助雨课堂等智慧教学平台实施课前自主学习、 课上交流互动、课后实践练习、单元学习检测等学 习阶段组成的全过程考评, 引导学生以自学为主、 听讲为辅的探究式学习。教学团队精心设计单元教 学运行体系, 包括遴选教学案例, 设计教学项目, 编制学习内容、题设、话题等, 根据课程单元的预 期学习产出设计成果评价体系; 适时评价学生在全 过程学习期间的各项学习表现及预期产出达成度。 以达成度为指引持续改进教学策略, 调整单元教学 运行体系的结构内容, 同时指导学生持续改进学习 
方法和策略, 为提升下一单元学习成就做准备。表 教学运行模型框架”示意表。 2 是“材料轧制成形工艺学”单元 CU01 的“OBE 单元 表 1. “材料轧制成形工艺学”课程单元与预期学习产出映射矩阵

\begin{tabular}{|c|c|c|c|c|c|c|c|c|c|c|c|c|c|c|}
\hline \multirow[b]{2}{*}{ 课程单元 } & \multicolumn{3}{|c|}{ 1.工程知识 } & \multicolumn{3}{|c|}{ 2. 问题分析 } & \multicolumn{3}{|c|}{3 设计/开发解决方案 } & & \multicolumn{3}{|c|}{ 4. 实验设计与信息处理 } & $\ldots$ \\
\hline & $\begin{array}{l}1.1 \text { 数 } \\
\text { 学 } \\
\text { 自然 } \\
\text { 科学 } \\
\text { 基础 } \\
\text { 知识 } \\
\\
\mathrm{ILO}_{1-} \\
1\end{array}$ & $\begin{array}{l}1.2 \text { 力 } \\
\text { 学 } \\
\text { 㫣学 } \\
\text { 等学 } \\
\text { 科基 } \\
\text { 础知 } \\
\text { 识 } \\
\mathrm{ILO}_{1-} \\
2\end{array}$ & $\begin{array}{l}1.3 \text { 材 } \\
\text { 料成 } \\
\text { 形原 } \\
\text { 理等 } \\
\text { 专业 } \\
\text { 基础 } \\
\text { 知识 } \\
\mathrm{ILO}_{1-} \\
3\end{array}$ & $\begin{array}{l}2.1 \text { 运 } \\
\text { 甹 } \\
\text { 程 } \\
\text { 知 } \\
\text { 别 识 } \\
\text { 表 达 } \\
\text { 工 程 } \\
\text { 问题 } \\
\mathrm{ILO}_{2-1}\end{array}$ & $\begin{array}{l}2.2 \text { 运 } \\
\text { 专 } \\
\text { 业知 } \\
\text { 识 分 } \\
\text { 题 }\end{array}$ & $\begin{array}{l}2.3 \text { 查 } \\
\text { 阅 } \\
\text { 献 分 } \\
\text { 析 问 } \\
\text { 题 }\end{array}$ & 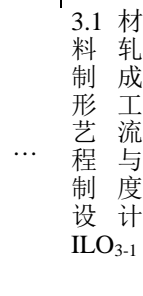 & $\begin{array}{l}3.2 \text { 材 } \\
\text { 料 轧 } \\
\text { 制成 } \\
\text { 形 设 } \\
\text { 备选 } \\
\text { 择 与 } \\
\text { 工具 } \\
\text { 设 计 } \\
\mathrm{ILO}_{3-2}\end{array}$ & $\begin{array}{l}3.3 \text { 基 } \\
\text { 于 } \text { 企 } \\
\text { 业 } \\
\text { 境 } \\
\text { 的 } \\
\text { 复 } \\
\text { 迥 } \\
\text { 解 } \\
\text { 方案 } \\
\mathrm{ILO}_{3-3}\end{array}$ & $\ldots$ & $\begin{array}{l}4.1 \text { 实 } \\
\text { 验研 } \\
\text { 究数 } \\
\text { 数 } \\
\text { 分 析 } \\
\text { 知 识 } \\
\text { 与 技 } \\
\text { 术 } \\
\mathrm{ILO}_{4-1}\end{array}$ & $\begin{array}{l}4.2 \text { 材 } \\
\text { 料 轧 } \\
\text { 制 成 } \\
\text { 形 实 } \\
\text { 验 仪 } \\
\text { 器 设 } \\
\text { 备操 } \\
\text { 作 } \\
\mathrm{ILO}_{4-2}\end{array}$ & $\begin{array}{l}4.3 \text { 基 } \\
\text { 于 汞 } \\
\text { 程问 } \\
\text { 题 的 } \\
\text { 实验 } \\
\text { 设计 } \\
\mathrm{ILO}_{4-} \\
2\end{array}$ & \\
\hline $\begin{array}{l}\text { 型材轧制成形 } \\
\text { 工艺 } \mathrm{CU}_{01}\end{array}$ & & & 3 & & 3 & 4 & 3 & & & & & & & \\
\hline $\begin{array}{l}\text { 板带材轧制成 } \\
\text { 形工艺 } \mathrm{CU}_{02}\end{array}$ & & & 4 & & 3 & 4 & 3 & & & & & & & \\
\hline $\begin{array}{c}\text { 板带材轧制工 } \\
\text { 艺制度设计 } \\
\mathrm{CU}_{03}\end{array}$ & & & 5 & & 4 & 5 & 4 & 4 & & & & & & \\
\hline $\begin{array}{c}\text { 板带材轧制成 } \\
\text { 形质量控制 } \\
\mathrm{CU}_{04}\end{array}$ & & & 6 & & 5 & 5 & 4 & & & & & & & \\
\hline $\begin{array}{l}\text { 管材轧制成形 } \\
\text { 工艺 } \quad \mathrm{CU}_{05}\end{array}$ & & & 4 & & 3 & 4 & 4 & & 4 & & & & & \\
\hline $\begin{array}{l}\text { 斜轧理论与变 } \\
\text { 形工具 } \mathrm{CU}_{06}\end{array}$ & & & 2 & & 3 & 4 & 4 & 4 & & & & & & \\
\hline $\begin{array}{c}\text { 管材纵轧理论 } \\
\text { 与变形工具 } \\
\text { CU }_{07}\end{array}$ & & & 3 & & 4 & 4 & 4 & 4 & & & & & & \\
\hline $\begin{array}{l}\text { 管材轧制成形 } \\
\text { 工艺制度 } \mathrm{CU}_{08}\end{array}$ & & & 4 & & 5 & 4 & 4 & & & & & & & \\
\hline $\begin{array}{l}\text { 材料轧制成形 } \\
\text { 实验 } \quad \mathrm{CU}_{09}\end{array}$ & & & 4 & 3 & & 4 & & & & & 5 & 6 & 4 & \\
\hline $\begin{array}{l}\text { 材料轧制成形 } \\
\text { 课题研究 } \mathrm{CU}_{10}\end{array}$ & & & 5 & 6 & 5 & 6 & & & 5 & & 6 & 6 & 5 & \\
\hline $\begin{array}{l}\text { 材料轧制成形 } \\
\text { 工程设计 } \mathrm{CU}_{11}\end{array}$ & & & 6 & 5 & 5 & 6 & 6 & 6 & 6 & & & & 5 & \\
\hline $\begin{array}{c}\text { “材料轧制成形 } \\
\text { 工艺学” } \\
\mathrm{CU}_{12}\end{array}$ & & & 5 & 5 & 5 & 6 & 4 & 5 & 6 & & 5 & 6 & 5 & \\
\hline
\end{tabular}

\section{3. 开展探究式课程单元教学}

以课程单元的预期学习产出为目标, 借助智慧课 堂教学平台, 使用 $\mathrm{QQ}$ 、微信等社交平台, 多维度跨 时空开展教学活动, 创造性地运用探究式教学方 法, 引导学生探究发现学习、探究项目学习, 促使 学生达成或提升学习产出。

材料成形及控制工程专业的很多专业知识不仅理 论性、逻辑性强, 更重要的是来源于、发展于、创 新于实验研究和工程实践, 单元教学不仅强化理 论, 也要强化实践, 更要理论联系实际, 探究式发 现教学法、探究式案例(项目)教学法教学效果更加显 著。[10-11]教学案例大多是知识面广、技术复杂的工 程实际事例, 案例问题的解决途径不唯一、分析结 论不确定, 需要全面综合运用本专业甚至跨专业的 学科知识和技术多维度、创造性思维, 全面分析问 题, 对比研究可行方案, 才可能得到科学的、合理
的、更优的分析结果, 得出相对正确的研究结论。 为此, 教学团队研究实践了一种探究式案例教学 法, ${ }^{[10]}$ 以学习产出为导向, 创设工程情境式教学案 例, 以探索发现式学习为手段进行课程单元教学。 材料成形及控制工程专业实践课程(或课程单元), 如 课程设计、综合/设计性实验、项目研究等, 是本专 业学生培养工程实践能力、探索创新能力、综合运 用专业知识解决复杂工程问题能力的最直接、最重 要的学习环节。为适应这类课程(或课程单元)的 OBE 教学需求, 教学团队将探究式、发现式、情境 式等教学方法融合为一体, 创立一种探究式发现教 学法。[11]针对教学课程(或课程单元)创立学习产出导 向的探究式发现教学运行体系, 设立学习小组, 组 建指导教师团队, 虚拟项目意境, 融合情境教学 法, 实施以组员为主体、组长为主导、教师为指导 的探究式教学, 引导学生探究学习、发现学习, 激 发求知欲望、求新意识, 提升探索能力, 促成自主 学习、合作创新。 
表 2. OBE 单元教学运行模型框架

课程: 材料轧制成形工艺学 教学单元: $\mathrm{CU}_{01}$ 教学策略: 探究式教学法

\begin{tabular}{|c|c|c|c|c|c|}
\hline ILO & 单元学习进程 & 学习成果展示项目 & 评价主体 & 评价指标 & ILO 达成度 \\
\hline \multirow{4}{*}{$\begin{array}{l}\mathrm{ILO}_{1-3}=3 \\
\mathrm{ILO}_{2-2}=3 \\
\mathrm{ILO}_{3-1}=4\end{array}$} & 课程自主学习 & $\begin{array}{l}\text { 预习, 课外研读 } \\
\text { 自学检测与练习 }\end{array}$ & $\begin{array}{l}\text { 教师评价 } \\
\text { 学生自评 } \\
\text { 学生互评 }\end{array}$ & $\begin{array}{l}\text { 学习热情, 信息检索能 } \\
\text { 力, 固旧知新能力等 }\end{array}$ & \multirow{4}{*}{$\begin{array}{l}\mathrm{ILO}_{1-3}=\omega_{1} \\
\mathrm{ILO}_{2-2}=\omega_{2} \\
\mathrm{ILO}_{3-1}=\omega_{3}\end{array}$} \\
\hline & 课上交流互动 & $\begin{array}{l}\text { 签到和随机点名 } \\
\text { 课堂互动(投稿、弹 } \\
\text { 幕) } \\
\text { 课堂适时测试 } \\
\text { 课堂讨论 }\end{array}$ & $\begin{array}{l}\text { 教师评价 } \\
\text { 学生自评 } \\
\text { 学生互评 }\end{array}$ & $\begin{array}{l}\text { 学习热情, 知识掌握, 知 } \\
\text { 识与技能的应用, 思维反 } \\
\text { 应能力, 团队协作能力, } \\
\text { 语言表达能力等 }\end{array}$ & \\
\hline & 课后实践练习 & $\begin{array}{l}\text { 课后大作业 } \\
\text { 课后工程实践练习 }\end{array}$ & $\begin{array}{l}\text { 团队评价 } \\
\text { 学生互评 }\end{array}$ & $\begin{array}{l}\text { 学习热情, 知识与技能的 } \\
\text { 应用, 团队协作能力, 工 } \\
\text { 程计算与分析能力, 实践 } \\
\text { 操作能力, 创新意识与能 } \\
\text { 力等 }\end{array}$ & \\
\hline & 单元学习测试 & $\begin{array}{l}\text { 标准化测试 } \\
\text { 非标准化测试 }\end{array}$ & $\begin{array}{l}\text { 教师评价 } \\
\text { 学生互评 }\end{array}$ & $\begin{array}{l}\text { 知识理解与掌握, 知识与 } \\
\text { 技能的应用, 思维反应能 } \\
\text { 力, 文字表达等 }\end{array}$ & \\
\hline
\end{tabular}

基于 $\mathrm{OBE}$ 理论的探究式案例教学和探究式发现 教学充分体现了以学生为中心、以产出为导向的教 学思想, 强调知识创新性和实践性, 引导学生通过 研究和实践来体验和发现知识, 进而构建和发展知 识, 而不是简单的接受和记忆知识; 培养学生创新 思维、开拓方法、灵活运用知识解决工程问题的能 力; 提升学生创新知识的意识和能力。

\section{4. 综合评价全过程学习产出}

基于 OBE 单元学习的考核形式多样、内容构成 复杂、难度不均, 特别是评价主体多元化, 评价尺 度和标准不统一, 传统的学习成绩分析方法不能准 确表达学生个体学习状态的差异, 评价结果不能反 映学生学习产出的真实性。为此, 教学团队研究并 实践一种基于信息理论的课程学习全过程考核成绩 分析算法(另文阐述)。运用该算法对学生单元学习全 过程考核产生的非标准化的、多元主体评判的、尺 度各异的多项成绩进行统一有效的等级评价。首先 将同一评价指标(预期学习产出)相关的考核成绩加权 合并, 运用聚类算法将全部学生的同一项学习产出 进行分类, 得到学习产出差异化等级评价; 运用摘 权法综合分析学生单元学习产出的综合表现, 达成 学习成就的形成性评价或终结性评价。学习全过程 考核成绩分析算法能做到评价适时、效度良好、信 度可靠, 实现差异化、个性化的学习评价, 突出自 我学习、自我提升的 OBE 评价理念。

\section{4. 结语}

随着国内高校教育改革深入发展, 为适应工程教 育专业认证需要, 众多高校开始接受并实践 OBE 教 学理念。作为 OBE 核心思想之一的学习产出评价方 法正逐渐成为教育学者和高校教师的研究重点。本 文研究了基于 OBE 教育理念的课程学习全过程非标
准化评价策略, 其实施程序如下:

（1）以学习产出为导向逆向设计教授课程, 运 用布卢姆目标分类法构建课程单元与学习产出映射 矩阵, 明确学习目标;

(2) 以学习产出为导向逆向设计教学单元, 以 探究式教学法为策略构建单元教学运行模型, 明晰 教学方法、教学内容、学习产出评价体系;

(3) 以学习产出为导向, 运用探究式案例教学 法和探究式发现教学法开展以自主学习为主、指导 为辅的单元教学活动, 培养学生创新意识和创造能 力;

（4）以学习产出为导向, 以个性化、差异化评 价为目标, 开展课程学习全过程非标准化考核, 运 用成绩聚类算法和熵权算法综合分析考核成绩, 实 现适时、可信、高效的学习产出形成性评价。

课程学习全过程非标准化评价策略秉承 OBE 的 “所有学生都是成功学习者”的理念, 通过评价对学 生进行结构性区分或分类, 有效地激励学生持续改 进, 逐步实现成功学习, 达成顶峰产出。

\section{致谢}

本文研究工作得到安徽省教育厅质量工程教育教 学研究重大项目“以能力为导向的非标准化学习评价 体系的研究与应用” (编号: 2018jyxm0016)、重大 项目 “非标准答案考试改革研究”(编号: 2018jyxm0952）子课题“以《塑性成型工艺(轧制)》 为例的学习效果评价方法的研究与应用” (编号: 2018jyxm0952-44)、省级教学团队项目“材料科学与 工程教学团队”（编号：2020jxtd266）资助, 在此表 示感谢。 


\section{REFERENCES}

[1] J. Sun, W.F. Wang. Exploration of nonstandardized examination mode in Colleges and Universities [J].Journal of Changzhi University, 2016.33(2):96-97(in Chinese)

[2] P.H. Gu, W.L. HU, P. Lin, etc. OBE engineering education model in Shantou University[J]. Research in Higher Education of Engineering, 2014,(1):27-37(in Chinese)

[3] Z.Y. Li, H. Zhu, Z.J. Liu, etc. The concept and cultivation of appied talent[J]. Research in Higher Education of Engineering, 2014,(2):29-35 (in Chinese)

[4] X.Q. Pei, W.J. Shen, H.R. Zhang. Discussion on the diversification of University course assessments[J].Higher Education in Chemical Engineering,2017,(5):53-57 (in Chinese)

[5] G.Z. Meng, Y. LU, J.N. Wang. Exploration and practice of engineering mathematics test model based on non-standardized questions[J]. Journal of Science of teachers' College and University, 2018,38(10):48-50(in Chinese)

[6] Q. wang, L.X. Song, W.C. Wei, etc. Teaching and learning platform and nonstandard examination system of computer general course in new engineering $[\mathrm{J}]$. Computer Education, 2019,(4):102-106(in Chinese)

[7] L.Li, X.M. Wang. Research on higher education assessment mode based on formative evaluation and information entropy theory[J]. Journal of Jilin Institute of Chemical Technology, 2018,35(2):811(in Chinese)

[8] Z. Chen, J. Liang,B. Xie. Exploration on the curriculum reform of non standardized examination under the background of engineering education professional certification [J]. Education modernization,2018,(36):110-111(in Chinese)

[9] J.B. Zhang, H.Y. Zhang, J. Liu. Application and practice on objective teaching method to professional courses of Material Forming and Control Engineering[J]. Journal of Xinxiang University,2014,31(2):74-77(in Chinese)

[10] J.B.Zhang, J.Liu, Z.Y.Duan, etc. The inquiry case teaching method in engineering technology courses computer \& network assisted teaching platform[C]. Processing of CICET2014,909-913

[11] J.B.Zhang, J.W.Wang, X.Q.Xu. The target-oriented inquiry-discovery teaching model in course design assisted by computer[C]. Processing of CICET2014,267-271 